\title{
Sleeping satellites: A risky prospect
}

\author{
Mark Tepfer and Mireille Jacquemond
}

Plants can be made resistant to virus infection by transformation with genes that express sequences from the target virus. Shortly after the first publications describing virus-resistant transgenic plants, it was pointed out that the use of certain viral genes could perhaps entail ecological risks ${ }^{1-3}$. The article by Palukaitis and Roossinck ${ }^{4}$ in this issue confirms that considerable risks can be associated with genes encoding satellite RNA (sat-RNA) that confer resistance to cucumber mosaic virus (CMV). Potential risks associated with CMV sat-RNA have been reviewed recently in detail ${ }^{5}$.

CMV is one of the most widely occurring plant viruses. It infects more than 800 plant species and has a considerable negative impact on agriculture worldwide. In addition to an RNA genome, certain strains of CMV naturally harbor an RNA of $330-400$ nucleotides, called sat-RNA, that depends on the virus for its entire cycle, has no significant similarity with the viral genome, and apparently does not encode protein. SatRNAs are capable of attenuating CMV strains, resulting in a sharp decrease in virus titer in infected plants and an almost complete lack of symptoms. Several groups have created plants that express sat-RNA genes and are thus highly tolerant to CMV infection $^{5}$. Unfortunately, some sat-RNAs-only in conjunction with certain hosts-exacerbate rather than attenuate symptoms. The best-known example is lethal tomato necrosis, epidemics of which have resulted in severe losses in southern Italy ${ }^{6}$ (see Fig. 1) and in Spain ${ }^{7}$. Other sat-RNAs also lead to stunting in tomato or pronounced mosaics in tomato or tobacco.

The sequence and secondary structure of CMV sat-RNAs are highly conserved, and thus sequence differences underlying symptom attenuation or worsening can be very slight. As shown in Figure 2, there are four known positions where a point mutation can transform an attenuating sat-RNA into one inducing lethal tomato necrosis. In addition, a point mutation at a different part

Mark Tepfer is directeur de recherche at the Laboratoire de Biologie Cellulaire, INRAVersailles, 78026 Versailles Cedex, France (mark@versailles.inra.fr) and Mireille Jacquemond is directeur de recherche at the Station de Pathologie Végétale, INRADomaine St. Maurice, BP 94, 84143 Montfavet Cedex, France (jacquemo@avi-

amp.avignon.inra.fr). of the sat-RNA leads to induction of a severe mosaic in tobacco $0^{4}$. Thus, the danger lies in

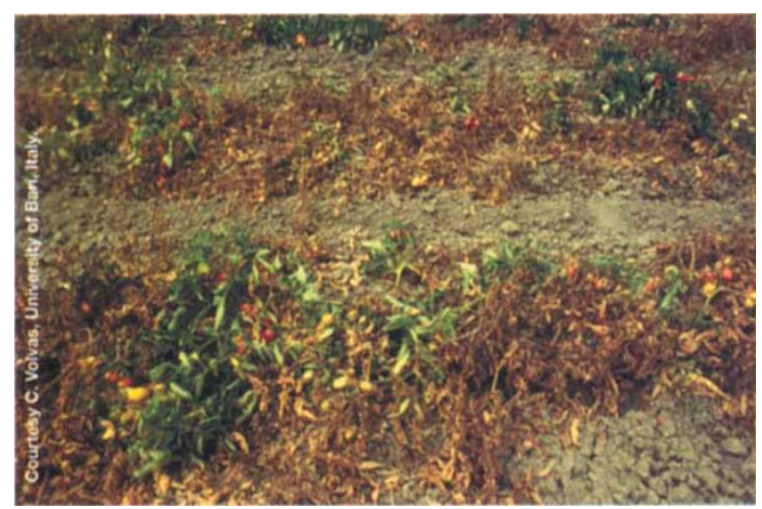

Figure 1. A tomato field in southern Italy devastated by lethal necrosis in 1988 by a strain of CMV with a necrogenic sat-RNA.

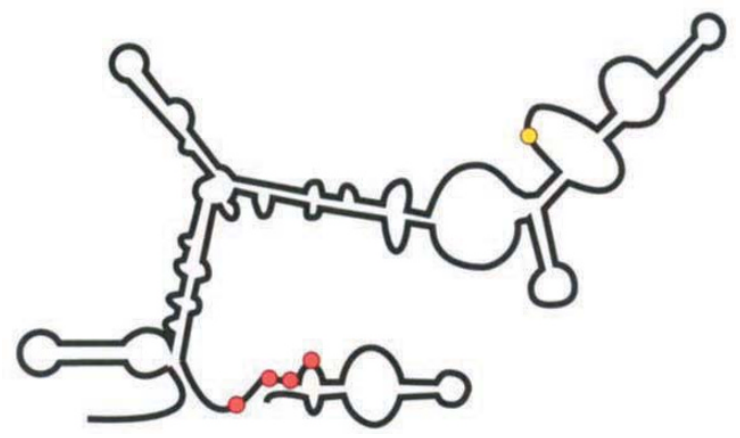

Figure 2. Structure of a typical CMV satellite RNA showing point mutations imparting deleterious effects (mutations leading to tomato necrosis are shown in red and the position involved in tobacco mosaic is shown in yellow).

the possibility that, even with a gene that encodes an entirely beneficial sat-RNA, on infection with CMV, the sat-RNA will be amplified to a high level, during which a point mutation can lead to a deleterious variant, which could then be transmitted to other host plants.

Although this sequence of events was expressed as a hypothesis some years ago, the fact that the appearance of deleterious forms of sat-RNA was a rare event in nature suggested that such mutations were unlikely to occur in transgenic plants. It was also reassuring that during several years of large-scale field cultivation in China of tobacco plants transformed with a sat-RNA gene, no deleterious effects were observed, either in the tobacco fields or in neighboring plants ${ }^{8}$. Now, Palukaitis and Roossinck have shown that a deleterious sat-RNA can arise by mutational drift, and that it can have a selective advantage over the parent sat-RNA species, becoming the dominant element in the sat-RNA population from which it arose.

Do the results of Palukaitis and Roossinck mean the end of projects for the commercial use of CMV sat-RNA genes? Although the experimental laboratory conditions they used may facilitate the appearance of sat-RNA variants relative to field conditions, their results are a clear warning of the gravity of the potential risks. One would be hard put to eliminate all possibilities of mutation to deleterious forms, because the nucleotides that are involved in tomato necrosis are different from those involved in tobacco yellow mosaic, and could well be different from those involved in tomato white leaf or tomato stunting. However, there is at least one possibility for creating safer sat-RNAs that might well be worth exploring. If the satRNA could be modified in a manner to prevent encapsidation, this would prevent its transmission to other plants, and could thus limit any new mutants to the initially infected plant. As other plant viruses are known to have genomes with precise, well-defined sites of initiation of encapsidation, it is not unreasonable to suppose that this is the case for CMV sat-RNAs, and that their modification could lead to biologically contained sat-RNAs of greatly reduced ecological risk.

1. Hull, R. 1991. pp. $443-457$ in Recognition and Response in Plant-Vinus interactions. (NATO ASI, Vol. H41.) Fraser, R.S.S. (ed.). Springer-Verlag, Berlin.

2. Palukaitis, P. 1991. pp. 140-162 in Risk Assessment in Genetic Engineering. Levin, $M$. and Strauss, $H$. (eds.). McGraw-Hill, New York.

3. Tepfer, M. 1993. Bio/Technology 11:1125-1132.

4. Palukaitis, P. and Roossinck, M. 1996. Nature Biotechnology 14:1264-1268

5. Jacquemond, M. and Tepfer, M. 1996. In Control of Plant Virus Diseases. Khetarpal, R.K., Koganezawa, H., and Hadidi, A. (eds.). APS Press, St. Paul, MN, in press.

6. Crescenzi, A., Barbarossa, L., Gallitelli, D., and Martell, G.P. 1993. Plant Dis. 77:28-33.

7. Jorda, C., Alfaro, A., Aranda, M.A., Moriones, E., and Garcia-Arenal, F. 1992. Plant Dis. 76:363-366.

8. Yie, Y. and Tien, P. 1993. Sem. Virol. 4:363-368. 